\title{
New spatiotemporal method for assessing video quality
}

\section{Woei-Tan Loh and David Boon Liang Bong*}

Faculty of Engineering, Universiti Malaysia Sarawak, 94300 Kota Samarahan, Sarawak, Malaysia

Email: lohwt91@yahoo.com

Email: davidblbong@yahoo.com

*Corresponding author

\begin{abstract}
The existence of temporal effects and temporal distortions in a video differentiate the way it is assessed from an image. Temporal effects and distortions can enhance or depress the visibility of spatial effects in a video. Thus, the temporal part of videos plays a significant role in determining the video quality. In this study, a spatiotemporal video quality assessment (VQA) method is proposed due to the importance of temporal effects and distortions in assessing video quality. Instead of measuring the frame quality on a frame basis, the quality of several averaged frames is measured. The proposed spatiotemporal VQA method is significantly improved compared with image quality assessment (IQA) methods applied on a frame basis. When combined with IQA methods, the proposed spatiotemporal VQA method has comparable performance with state-of-the-art VQA methods. The computational complexity of the proposed temporal method is also lower when compared with current VQA methods.
\end{abstract}

Keywords: video; frames; video quality; spatial effects; temporal effects; temporal distortions; spatiotemporal; average; video quality assessment; VQA; image quality assessment; IQA; computational complexity.

Reference to this paper should be made as follows: Loh, W-T. and Bong, D.B.L. (2018) 'New spatiotemporal method for assessing video quality', Int. J. Computational Vision and Robotics, Vol. 8, No. 3, pp.336-348.

Biographical notes: Woei-Tan Loh received his BS in Electronics and Telecommunication Engineering from the Universiti Malaysia Sarawak, Malaysia in 2014. He is currently working towards Master of Engineering in the Universiti Malaysia Sarawak.

David Boon Liang Bong received his $\mathrm{PhD}$ from the Universiti Sains Malaysia, his Master of Science from the Nanyang Technological University, Singapore and his Bachelor of Electrical Engineering from the University of Technology, Malaysia. He is currently a Senior Lecturer with the Universiti Malaysia Sarawak. His research interests include visual quality assessment, feature extraction, computer vision, medical imaging and intelligent systems. 